\title{
A new sample of red supergiants in the Inner Galaxy
}

\author{
M. Messineo ${ }^{1}$, Q. Zhu ${ }^{1}$, D. F. Figer ${ }^{2}$, K. M. Menten ${ }^{3}$, V. D. Ivanov ${ }^{4}$, \\ R.-P. Kudritzki ${ }^{5}$ and C.-H. Rosie Chen ${ }^{3}$ \\ ${ }^{1}$ Key Laboratory for Researches in Galaxies and Cosmology, University of Science and \\ Technology of China, Chinese Academy of Sciences, Hefei, Anhui, 230026, China \\ ${ }^{2}$ Center for Detectors, Rochester Institute of Technology, 54 Memorial Drive, Rochester, NY \\ 14623, USA \\ ${ }^{3}$ Max-Planck-Institut für Radioastronomie, Auf dem Hügel 69, D-53121 Bonn, Germany \\ ${ }^{4}$ European Southern Observatory, Karl Schwarzschild-Strasse 2, D-85748 Garching bei \\ Munchen, Germany \\ ${ }^{5}$ Institute for Astronomy, University of Hawaii, 2680 Woodlawn Drive, Honolulu, HI 96822
}

\begin{abstract}
We carried out a pivot experiment to select distant luminous late-type stars on the basis on their 2MASS and GLIMPSE photometry. Low-resolution infrared spectra enabled us to measure the equivalent widths $(E W s)$ of their CO band-heads at $2.293 \mu \mathrm{m}$, and to confirm an extraordinarily high detection rate of red supergiants (RSGs), .i.e. 61\% (Messineo et al. (2016)).
\end{abstract}

Keywords. Galaxy: disk, stars: late-type, supergiants

\section{Overview of our survey}

RSGs are an important probe of Galaxy formation and evolution. Star formation is coupled with the Galactic potential and occurs in preferential locations, such as the two end sides of the Bar where a large number of RSGs have been detected. These locations contain an extraordinary number of RSGs that are easily detectable. Inspired by these findings, we tried to detect individual RSGs, independently of clusters.

Approximately one hundred targets were selected from the 2MASS and GLIMPSE North I surveys, by following the prescriptions of Messineo et al. (2012) with $Q 1$ and $Q 2$ extinction-free colors. We selected stars with $0.1<Q 1<0.5 \mathrm{mag}$ and $0.5<Q 2<1.5$ mag. This range includes about $46 \%$ of known RSGs (Messineo et al. (2012)). Lowresolution $H K$ spectra were collected with the SofI spectrograph on the ESO-NTT 4mtelescope. The $E W$ of the $C O$ band-head at $2.293 \mu \mathrm{m}$ is a good indicator of temperature. Giants and supergiants follow two distinct relations, and late-type RSGs have large $E W$ s. Contaminating AGB Miras can be classified by the shape of their continuum that is affected by broad $\mathrm{H}_{2} \mathrm{O}$ absorption. The spectroscopic analysis has resulted in an extraordinarily large number of new RSGs, obtaining a detection rate of $>60 \%$.

Distances vary from 3.6 to $8.6 \mathrm{kpc}$; they were estimated with surrounding clump stars (primary indicators of distance) by deriving a relation between reddening and distance for a given line-of-sight. Luminosities confirm that the sample is dominated by RSGs with initial masses from 12 to $20 M_{\odot}$. In conclusion, we successfully searched for RSGs and we increased by about $25 \%$ the number of previously known RSGs with $|b|<1^{\circ}$ and $10^{\circ}<l<65^{\circ}$. Only about $1.5 \%$ of these RSGs are found in clusters.

\section{References}

Messineo, M., Menten, K. M., Churchwell, E., \& Habing, H. 2012, A\&\&A, 537, A10

Messineo, M., Zhu, Q., Menten, K. M., et al. 2016, ApJL, 822, L5 